\title{
Rotational Atherectomy in Sub-Acute Anterior STEMI with Cardiogenic Shock
}

\section{Zaki Horizon Islami (D) Hendry Purnasidha Bagaswoto Nahar Taufiq \\ Budi Yuli Setianto}

Department of Cardiology and Vascular Medicine, Faculty of Medicine, Public Health and Nursing, Universitas Gadjah Mada - Dr. Sardjito Hospital, Yogyakarta, Indonesia
Correspondence: Hendry Purnasidha Bagaswoto

Department of Cardiology and Vascular Medicine, Faculty of Medicine, Public Health and Nursing, Universitas Gadjah Mada - Dr. Sardjito Hospital, Yogyakarta, Indonesia

Tel +6281904245266

Fax +62274547783

Email arsyadhiracarissa@gmail.com

\begin{abstract}
Rotational atherectomy (RA) is a proven technique to modify a heavily calcified coronary lesion if balloon angioplasty failed. RA is frequently avoided in ST-elevation myocardial infarction (STEMI) as it may increase the risk of slow or no-reflow. It is also considered to be relatively contraindicated in lesions with a visible thrombus, by its manufacturer. Regardless, RA may be a life-saving procedure in cases where no other percutaneous coronary intervention (PCI) technique is available to modify the lesion adequately. This case reports successful use of RA to facilitate dilation and stent delivery of a previously non-dilatable lesion in a patient with sub-acute anterior STEMI complicated by cardiogenic shock.
\end{abstract}

Keywords: rotational atherectomy, heavily calcified culprit lesion, STEMI, cardiogenic shock

\section{Background}

Rotational atherectomy (RA) is an endovascular procedure used to modify atherosclerotic plaque using a rotating burr. It was introduced approximately 30 years ago as an addition to standard balloon angioplasty which sometimes fails to dilate a lesion. ${ }^{1}$ RA had a usage rate as high as $10 \%$ in all percutaneous coronary intervention (PCI) procedures in the 1990s and showed a high success rate. ${ }^{2}$ Despite its early success, it has been associated with high rate of complications such as restenosis, which led to a marked decline in the use of this procedure to $3-5 \%{ }^{1}$

Half of patients with ST-elevation myocardial infarction (STEMI) who underwent PCI had a moderate to severely calcified lesion. ${ }^{3}$ Although RA was relatively contraindicated in acute coronary syndrome (ACS) settings due to its prothrombotic state, recent reports showed a decent procedural success with no significant difference in complications compared to RA procedure performed in stable patients. ${ }^{4,5}$ Consensus has recommended specific techniques regarding RA procedure to minimize complications. ${ }^{6,7}$ However, there are no current clinical guidelines available on performing RA in STEMI cases as its use has been a longstanding controversy.

\section{Case Presentation}

A 62-year-old man presented with typical chest pain lasting an hour since its acute onset when he was brought to a district hospital. The patient was diagnosed with anterior STEMI and given a loading dose of aspirin and clopidogrel. Unfortunately, 
the patient suffered a cardiac arrest afterwards due to ventricular fibrillation. After being successfully resuscitated, reperfusion therapy was planned however it was not possible due to a logistic problem at the district hospital. The patient was subsequently treated with an anticoagulant, statin, and morphine at the intensive care unit. However, his condition worsened, and he had recurrent chest pain. The patient was then referred to our hospital with anterior STEMI with onset $>24$ hours.

Upon arrival, his electrocardiogram (ECG) showed significant ST-segment elevation in anterior leads with right bundle-branch block (RBBB) (Figure 1). Subsequently, the patient underwent an emergency cardiac catheterization. Diagnostic coronary angiography demonstrated a total occlusion at the proximal left anterior descending (LAD) artery with suspected severely calcified lesion due to its dense radiopacity (Figure 2A). Mach 1 $1^{\mathrm{TM}}$ CLS 3.5/6F (Boston Scientific Corp., MA) Guide Catheter (GC) was engaged to cannulate left coronary artery (LCA) with Hi-Torque Balance Middleweight Universal II (BMW) 0.014" wire (Abbott Vascular, Chicago, IL) used to cross the occluded lesion. Sprinter Legend 2.0" (Medtronic Corp., Minneapolis, $\mathrm{MN}$ ) and non-compliant (NC) Sprinter 2.75" (Medtronic Corp., Minneapolis, MN) Balloon Catheter (BC) were simultaneously used to dilate the lesion. The flow to distal LAD was restored with TIMI-2 flow; however, leaving $90 \%$ residual stenosis at the primary lesion (Figure 2B). Multiple attempts to modify the lesion failed, starting with double wire technique with $\mathrm{Hi}$ Torque PILOT 50 0.014" (Abbott Vascular, Chicago, IL) wire to provide more support then followed by balloon dilatation with Scoreflex ${ }^{\circledR}$ NC scoring 2.0" (OrbusNeich, Hong Kong) and other mentioned BC. Due to inability to properly dilate the calcified lesion, an attempt to insert a stent was abandoned and the procedure was ended with TIMI-2 flow result.

On the following day, the patient had ongoing chest pain and his blood pressure dropped. Inotropes and a small dose of vasoconstrictor drugs were administered to reach a mean arterial pressure (MAP) above 65. Intra-aortic balloon pump (IABP) was also inserted to provide extra hemodynamic support. After careful consideration regarding the unstable hemodynamic condition of this patient, another intervention was planned to improve the clinical condition of the patient. Re-PCI with RA was done with the intention to debulk the calcified lesion so a complete stent deployment could be achieved. Re-PCI was preferred since our hospital was not capable of doing urgent coronary artery bypass graft (CABG) operations.

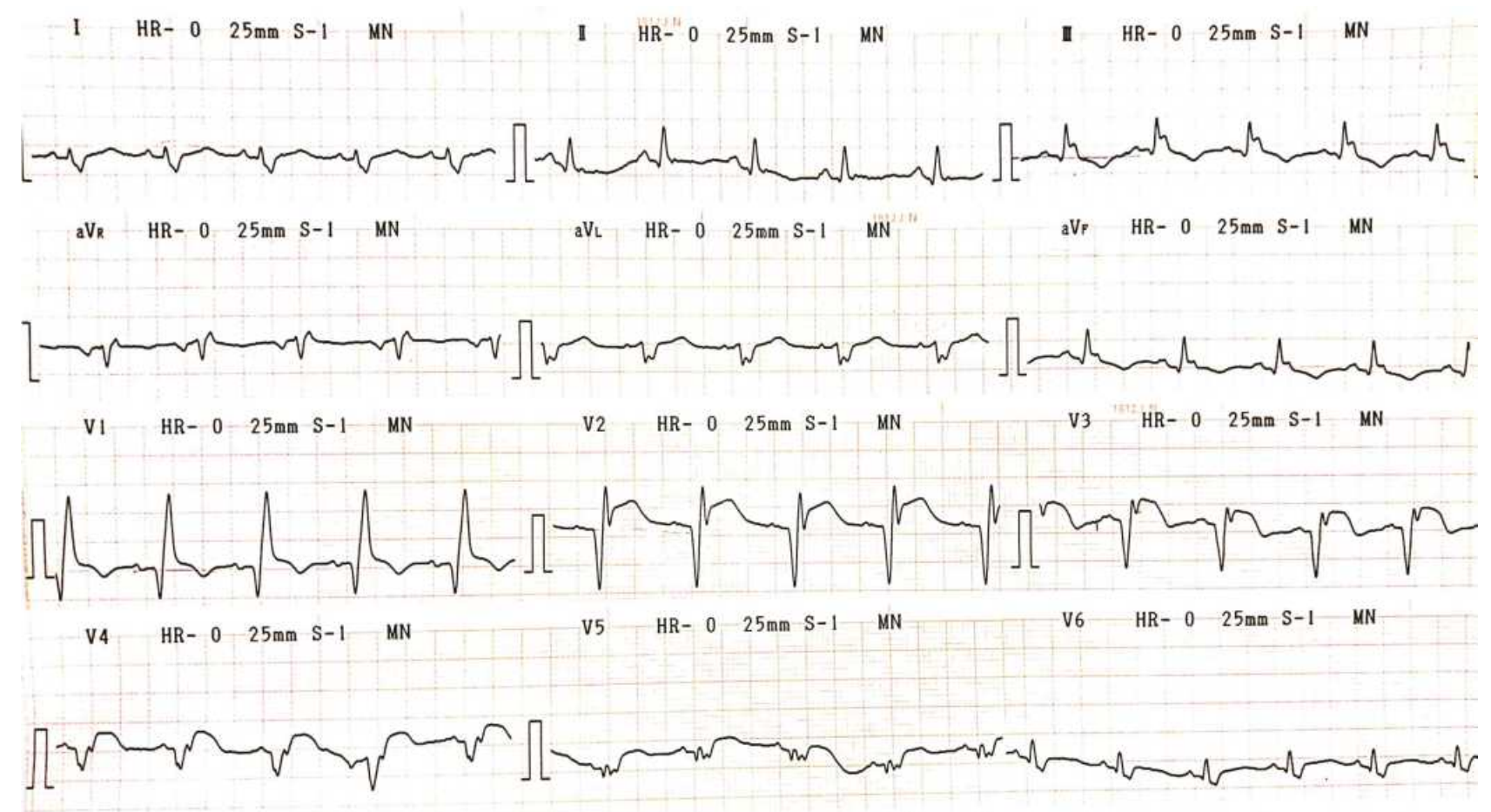

Figure I Electrocardiogram showed ST-segment elevation in anterior leads with right bundle-branch block pattern. 


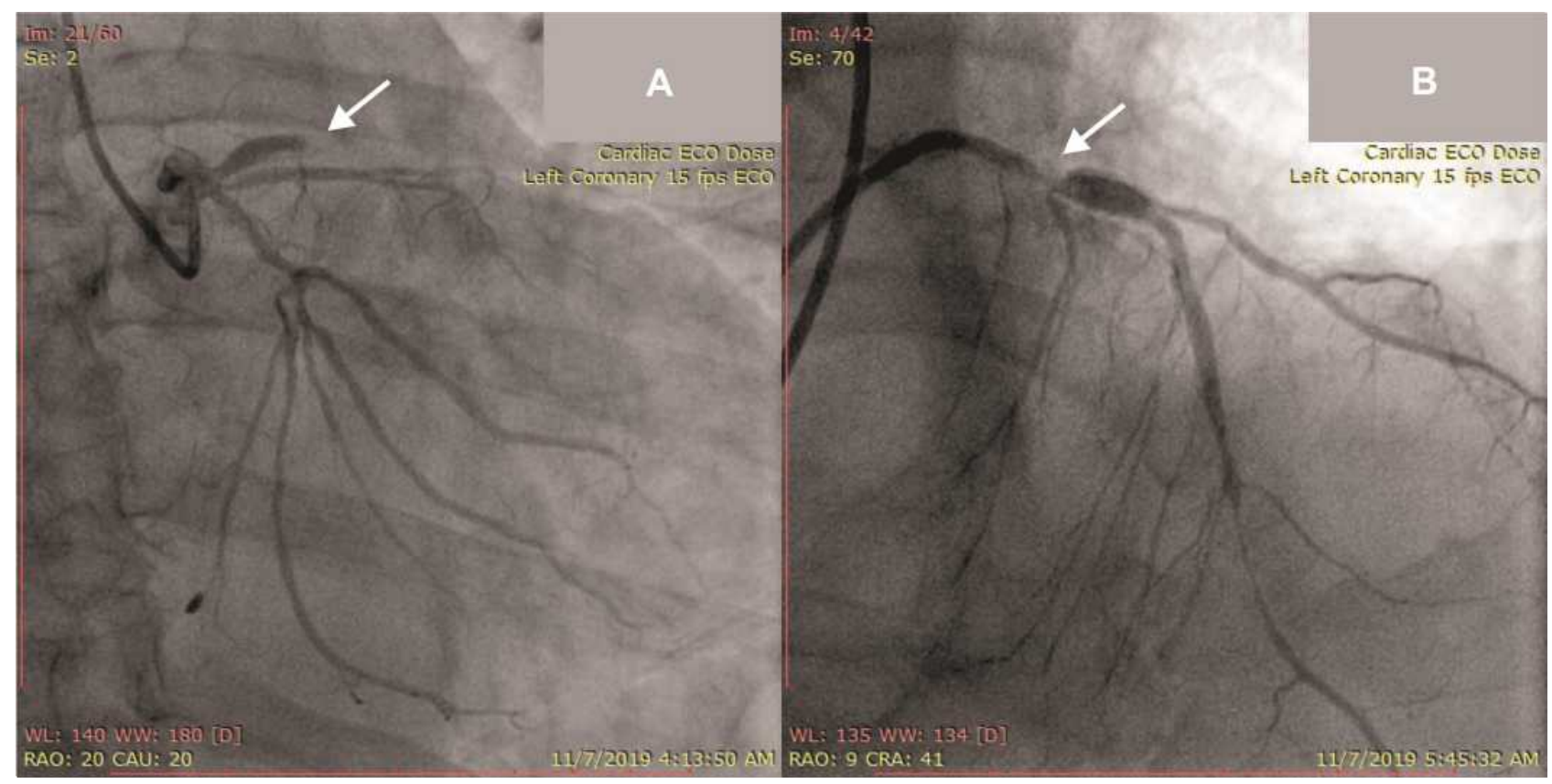

Figure 2 (A) Total occlusion at proximal segment of left anterior descending artery from coronary angiography pre-primary PCl (arrow); (B) final result after primary PCl showing significant residual stenosis at proximal left anterior descending artery (arrow).

Angiography evaluation revealed the residual stenosis at main LAD lesion (Figure 3A). Mach 1 ${ }^{\mathrm{TM}}$ CLS 3.5/7F (Boston Scientific Corp., MA) GC was used to cannulate LCA. Several guide wires failed to cross the lesion, and then we decided to use Fielder XT 0.014" which successfully crossed the lesion. Micro-Guide Catheter Finecross ${ }^{\circledR}$ (Terumo Corp., Tokyo) was used to facilitate insertion of Rota Wire 0.009" (Boston Scientific Corp., MA). Rota burr 1.75 (Boston Scientific Corp., MA) was used and RA was completed in modifying the calcified lesion with five subsequent pecking-motion runs at 160,0000-215,000 rpm for 8-10 seconds (Figure 3B). A bigger balloon, NC Sprinter 3.0 (Medtronic Corp., Minneapolis, $\mathrm{MN}$ ), was used to dilate before drug-eluting stents (DES) were placed (Figure 3C).

DES Xience Prime LL 3.5/33 mm (Abbott Vascular, Chicago, IL) was implanted at the proximal-mid-LAD with nominal pressure. DES Endeavor Resolute RX 2.75/ $30 \mathrm{~mm}$ (Medtronic Corp., Minneapolis, MN) was implanted at distal left circumflex artery (LCx) with nominal pressure. DES Xience Prime LL 3.5/28 mm (Abbott Vascular, Chicago, IL) was placed at ostial left main (LM) up to mid-LAD with nominal pressure (overlapped with previous LAD stent). T-stent technique was then performed at LM-LCx with DES Endeavor Resolute RX 3.0/18 mm (Medtronic Corp., Minneapolis, MN) with nominal pressure. Post-dilatation with proximal optimization technique was done using NC Sprinter balloon size 3.0 and ex-stent balloon size 3.0 both with nominal pressure. Final angiography after several stents were placed showed no residual stenosis, (Figure 3D) and no complication was observed during and after the procedure.

The patient's condition gradually improved after atherectomy was done, the chest pain was gone, and he was discharged after 10 days of hospitalization. The patient suffered heart failure due to delayed revascularization. Unfortunately, 7 months later, the patient was reported to have had cardiac arrest at home and passed away.

\section{Discussion}

Rotational atherectomy (RA) is a proven procedure to modify coronary lesion and to facilitate stent deployment in heavily calcified lesion if high-pressure balloon angioplasty alone failed to dilate. ${ }^{7}$ A heavily calcified lesion may destroy the drug-coated layer of a stent, thus lowering stent effectiveness due to uneven drug distribution. ${ }^{8}$ RA was the only PCI technique available in modifying fibrotic or heavily calcified culprit lesion in our center. It is a class IIA recommendation in ESC/EACTS myocardial revascularization guidelines. ${ }^{9}$

However, due to its highly invasive nature, RA may increase complication risk. RA procedure activated 


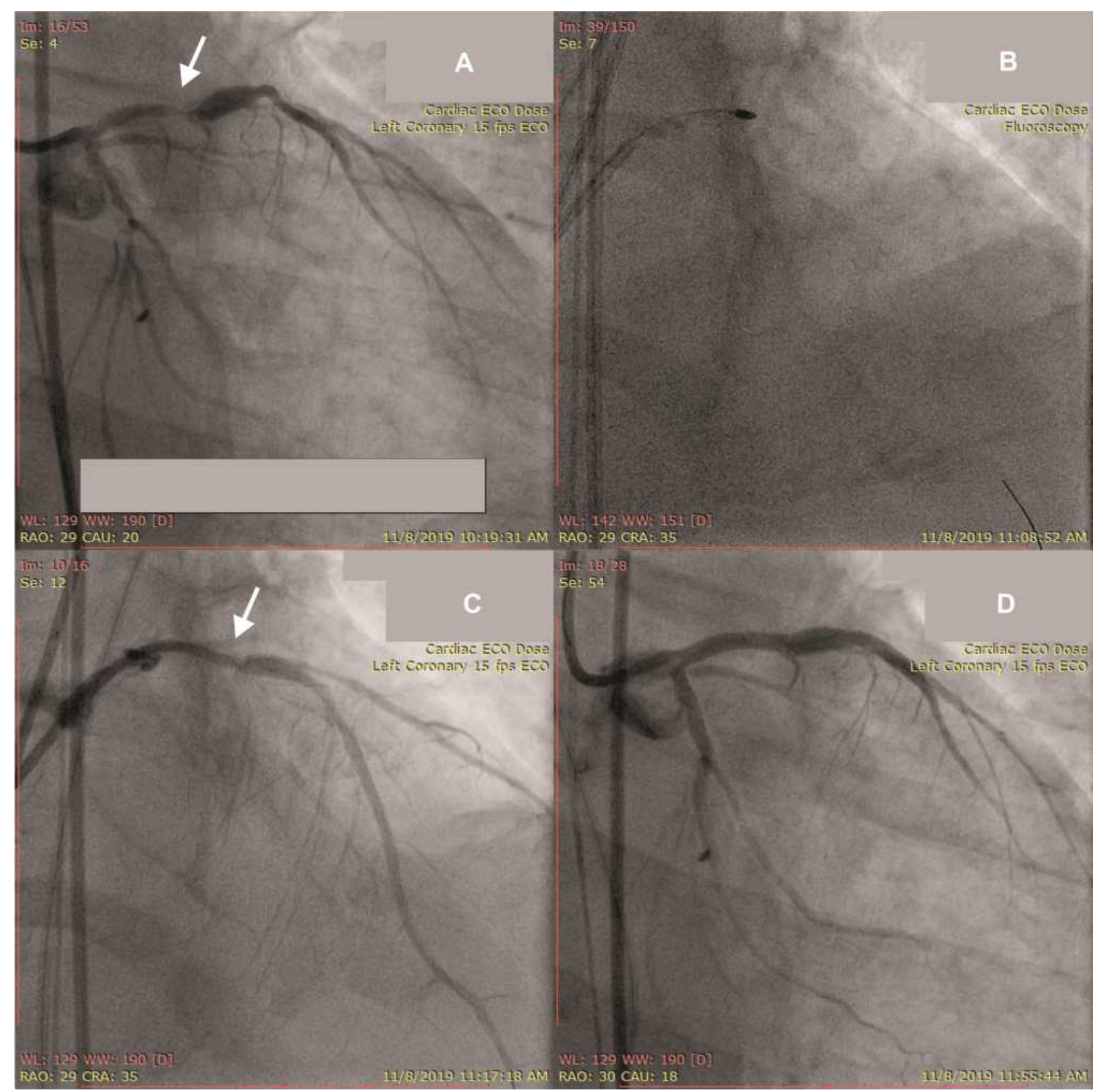

Figure 3 (A) Baseline cine pre-second PCl showing significant residual stenosis (arrow); (B) Rota burr I.75 was used to modify residual stenosis; (C) modified lesion after rotational atherectomy before stenting (arrow); (D) final result of second $\mathrm{PCl}$ (after stenting).

platelet aggregation as a result of opened coronary lesion after being modified. ${ }^{10}$ One of the absolute contraindications for RA mentioned by the manufacturer is presence of coronary artery thrombus. ${ }^{1}$ Despite the fact that no recommendations have been published yet, there have been several cases and studies assessing its use in the acute coronary syndrome (ACS) setting. According to pooling analysis from HORIZONS-AMI and ACUITY studies, $54-63 \%$ of STEMI patients were found to have moderate to severely calcified coronary lesions. ${ }^{3}$
In our case, the patient was unsuccessfully managed in the primary PCI attempt, with significant residual stenosis due to its heavy calcification. Since this result may jeopardize the patient's outcome with evidence of the presence of recurrent pain and hemodynamic instability, performing RA was unavoidable. Fortunately, when angiography was reperformed, the angiography did not show thrombus at residual lesion, thus, the RA procedure was expected to be carried out safely. This procedure proved to be successful, and there were no adverse consequences. 
Some retrospective studies showed rotational atherectomy in ACS had a high procedural success rate and no difference in survival rate nor major cardiac event (MACE) in long-term follow up versus RA done in nonACS patients. Wang et al (2017) reported RA procedure in acute myocardial infarct was a feasible option due to an effective and high procedural success rate with comparable clinical outcome (survival and MACE) in 30 days and one year follow up, compared to RA procedure in recent myocardial infarct patients. ${ }^{4}$ Mahmood et al (2017) also mentioned RA used in primary PCI settings as a safe option in a complex coronary lesion in which conventional balloon angioplasty failed to modify the lesion. ${ }^{11}$ Kubler et al (2018) also reported a similar result comparing RA in ACS patients against RA in stable angina patients with comparable survival rate and MACE in 1 year follow up. Thus, they encouraged RA procedure in ACS cases in which PCI cannot be delayed. ${ }^{5}$ However, special considerations must be carefully assessed since RA has been associated with higher post-procedural complications (bleeding at access site and contrast-induced nephropathy) and longer procedural time due to its complex preparation. $^{5,11}$

\section{Conclusion}

Rotational atherectomy (RA) is one of the techniques used in a heavily calcified lesion when balloon angioplasty has failed. It can be considered as an alternative option during PCI in STEMI settings. However, one should consider the benefits and disadvantages before commencing RA in acute STEMI settings due to its highly invasive nature which may increase the likelihood of procedural complications.

\section{Consent}

Written informed consent for publication of their details was obtained from the daughter of the patient. Institutional approval was not required to publish this case report.

\section{Disclosure}

The authors report no conflicts of interest in this work.

\section{References}

1. Tomey MI, Kini AS, Sharma SK. Current status of rotational atherectomy. JACC Cardiovasc Interv. 2014;7(4):345-353. doi:10.1016/j.jcin.2013.12.196

2. Lasala JM, Reisman M. Rotablator plus stent therapy (rotastent). Curr Opin Cardiol. 1998;13(4):240-247. doi:10.1097/00001573199807000-00004

3. Généreux P, Madhavan MV, Mintz GS, et al. Ischemic outcomes after coronary intervention of calcified vessels in acute coronary syndromes. J Am Coll Cardiol. 2014;63(18):1845-1854. doi:10.1016/j.jacc.2014.01.034

4. Wang TJ, Chiang MH, Huang SS, et al. Clinical outcomes of percutaneous coronary intervention with rotablation in patients with acute or recent myocardial infarction. J Chin Med Assoc. 2017;80 (9):532-538. doi:10.1016/j.jcma.2017.02.009

5. Kübler P, Zimoch W, Kosowski M, Tomasiewicz B, Telichowski A, Reczuch K. Acute coronary syndrome - still a valid contraindication to perform rotational atherectomy? Early and one-year outcomes. J Cardiol. 2018;71(4):382-388. doi:10.1016/j.jjcc.2017.10.012

6. Barbato E, Carrié D, Dardas P, et al. European expert consensus on rotational atherectomy. EuroIntervention. 2015;11(1):30-36. doi:10.4244/EIJV11I1A6

7. Sharma SK, Tomey MI, Teirstein PS, et al. North American expert review of rotational atherectomy. Circ Cardiovasc Interv. 2019;12(5): e007448. doi:10.1161/CIRCINTERVENTIONS.118.007448

8. Abdel-Wahab M, Richardt G, Joachim Büttner H, et al. High-speed rotational atherectomy before paclitaxel-eluting stent implantation in complex calcified coronary lesions: the randomized ROTAXUS (Rotational atherectomy prior to taxus stent treatment for complex native coronary artery disease) trial. JACC Cardiovasc Interv. 2013;6 (1):10-19. doi:10.1016/j.jcin.2012.07.017

9. Neumann FJ, Sousa-Uva M, Ahlsson A, et al. 2018 ESC/EACTS Guidelines on myocardial revascularization. Eur Heart J. 2019;40 (2):87-165. doi:10.1093/eurheartj/ehy394

10. Iannaccone M, Piazza F, Boccuzzi GG, et al. RoTational AThErectomy in acute coronary syndrome: early and midterm outcomes from a multicentre registry. EuroIntervention. 2016;12 (12):1457-1464. doi:10.4244/EIJ-D-15-00485

11. Mahmood M, Qureshi M, Morley R, et al. 27 Use of rotational atherectomy in primary pci for st-elevation myocardial infarctiona single centre 10-year experience. Heart. 2017;103(Suppl 5):A22A23. doi:10.1136/heartjnl-2017-311726.27
International Medical Case Reports Journal

\section{Publish your work in this journal}

The International Medical Case Reports Journal is an international, peer-reviewed open-access journal publishing original case reports from all medical specialties. Previously unpublished medical posters are also accepted relating to any area of clinical or preclinical science. Submissions should not normally exceed 2,000 words or 4 published pages including figures, diagrams and references. The manuscript management system is completely online and includes a very quick and fair peer-review system, which is all easy to use. Visit http://www.dovepress.com/testimonials.php to read real quotes from published authors. 